\title{
Distinct or Gradually Changing Spatial and Nonspatial Representations along the Dorsoventral Axis of the Hippocampus
}

\author{
Michael M. Yartsev \\ Department of Neurobiology, PhD Program, Weizmann Institute of Science, Rehovot 76100, Israel \\ Review of Royer et al.
}

The hippocampus provides neuroscientists with one of the most striking neural phenomena in any high-order brain area: the encoding of the animal's self location. As the animal moves through the environment, individual hippocampal pyramidal cells become active, mainly when the animal passes through a restricted area of the environment; these neurons are called "place cells," and the region of the environment where they are active is called the "place field." Since the discovery of place cells almost 40 years ago (O'Keefe and Dostrovsky, 1971), the majority of electrophysiological studies of their function focused on its dorsal third, since in the rodent this part is experimentally the most accessible (Andersen et al., 2007). It is widely accepted that the dorsoventral axis of the hippocampus (also known as the septotemporal, or longitudinal, axis) is not uniform in its inputs. The more dorsal parts receive mainly visuospatial information and the more ventral parts receive mainly nonspatial information related to reward and emotional stimuli, suggesting that different levels of this axis

Received April 8, 2010; revised May 4, 2010; accepted May 6, 2010.

M.M.Y. is supported by the Feinberg Graduate School of the Weizmann Institute of Science and the Lev Zion Excellence Fellowship. I thank Nachum Ulanovsky and Etan Markus for helpful comments on this manuscript.

Correspondence should be addressed to Michael M. Yartsev, PhD Student, Department of Neurobiology, Weizmann Institute of Science, Rehovot 76100, Israel. E-mail: myartsev@gmail.com.

DOI:10.1523/JNEUROSCI.1787-10.2010

Copyright $\odot 2010$ the authors $\quad 0270-6474 / 10 / 307758-03 \$ 15.00 / 0$ might subserve different functions. However, support for this idea comes mainly from neuroanatomical studies; very few electrophysiological studies systematically tested differences along the dorsoventral axis, especially at its ventral most tip (Andersen et al., 2007). A recent study published in the Journal of Neuroscience (Royer et al., 2010) was designed to tackle this shortcoming and explore differences between dorsal and ventral hippocampus in the rat. The authors recorded the activity of single neurons from the dorsal and ventral parts of hippocampal area CA3 during various behavioral tasks [Royer et al. (2010), their Fig. 1], which allowed them to look for neural correlates that might vary between the two regions. In turning to discuss the methodology, results, and our interpretations of the study by Royer et al. (2010), we will focus on three main topics: (1) spatial representation; (2) role of the theta oscillation; and (3) coding of nonspatial information such as fear/stress and reward in ventral hippocampus.

First, using a task commonly used for studying hippocampal place cells, foraging in a two-dimensional open field, the authors found that ventral neurons lacked the spatial specificity that is the hallmark of dorsal hippocampal neurons. They showed that unlike dorsal neurons, which are active in a small area of the environment, ventral neurons usually fired at multiple locations and sometimes over the entire arena [Royer et al. (2010), their Fig. 2]. Royer et al. (2010) hence suggested that the two regions fundamentally differ in their capacity to encode the animal's location. Yet, these results cannot rule out the possibility that ventral hippocampal neurons code for the animal's location on a larger spatial scale-larger than the arena size used in their study $(1 \times 2 \mathrm{~m})$. Indeed, previous studies have shown that the size of place fields increases along the dorsoventral axis (Fig. 1A) (Jung et al., 1994; Maurer et al., 2005; Kjelstrup et al., 2008). Furthermore, a recent study (Kjelstrup et al., 2008) showed that when using a standard two-dimensional arena of similar size to the one used by Royer et al. (2010), little spatial specificity was exhibited by ventral neurons; but, when the same neurons were recorded on a long $18 \mathrm{~m}$ linear track, their spatially specific firing became evident, and the authors found extremely large place fields with a mean length of $6.6 \mathrm{~m}$, much longer than the arena dimensions used by Royer et al. (2010).

Second, Royer et al. (2010) found that the power of theta oscillation (a prominent hippocampal local field potential rhythm) and the fraction of thetamodulated neurons were substantially reduced in ventral hippocampus (Fig. 1B) [Royer et al. (2010), their Fig. 6]. Furthermore, the authors found a correlation between the decrease in spatial specificity and theta modulation of ventral neurons [Royer et al. (2010), their Fig. 7]. These 
A

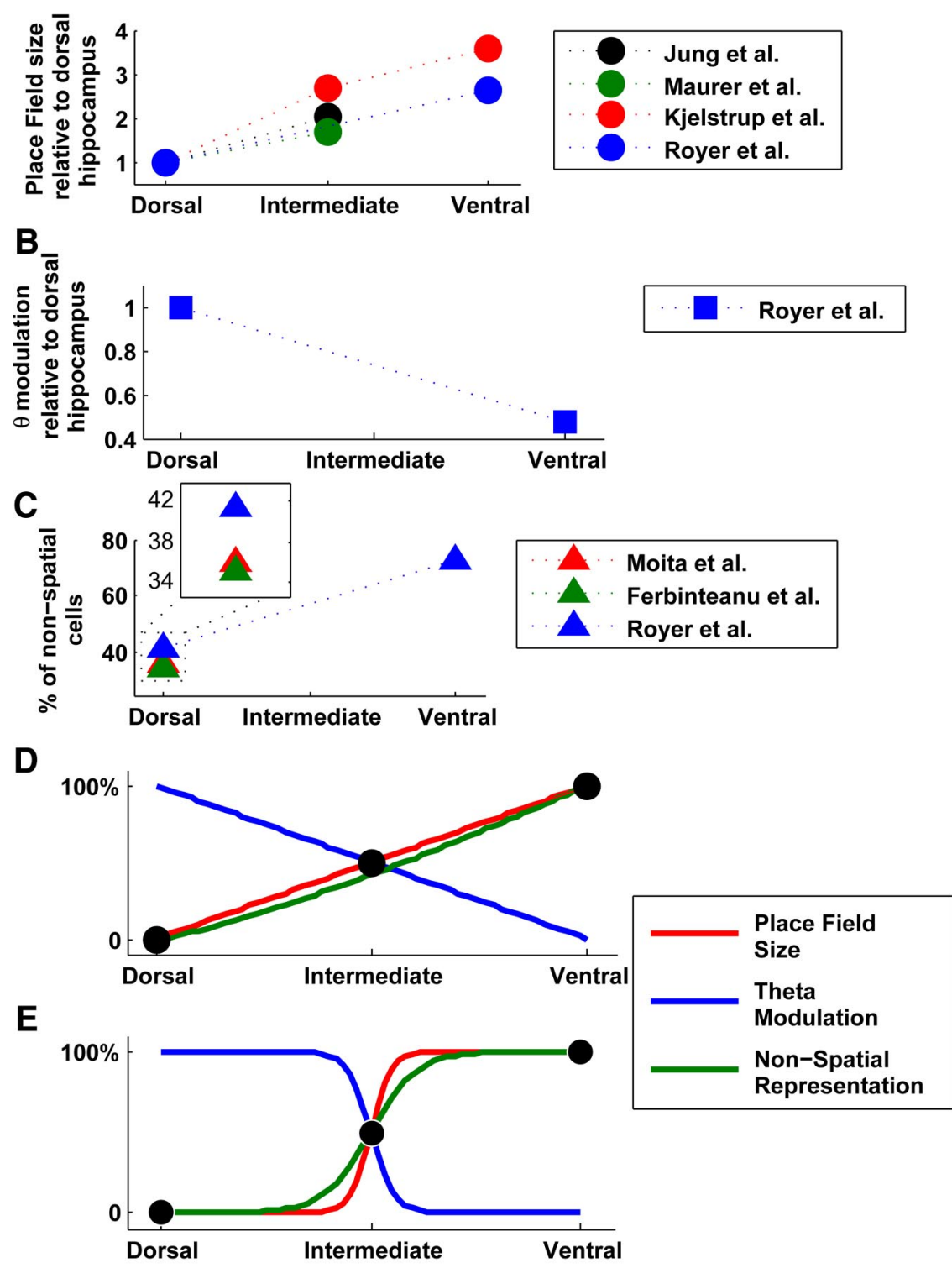

Figure 1. Properties of place-field size, theta oscillation, and nonspatial responses along the dorsoventral axis of the hippocampus. A, Summary of data from different studies (Jung et al., 1994; Maurer et al., 2005; Kjelstrup et al., 2008; Royer et al., 2010) showing the ratio of place-field size in dorsal, intermediate, and ventral hippocampus relative to the mean dorsal placefield size reported at each study. By this definition, the $y$-values for "Dorsal" are all normalized to 1 . For Jung et al. (1994), the values were computed as the square root of the two-dimensional place-field size. For Maurer et al. (2005) and Kjelstrup et al. (2008), values were taken directly from the reported place-field sizes on the linear track. For Royer et al. (2010), values were extracted from their Figure $2 B$ as the percentage of active pixels in the dorsal and ventral cells. $B$, Ratio of the theta modulation of neurons in ventral versus dorsal hippocampus, computed from the mean theta index of ventral and dorsal pyramidal cells, extracted from Figure $6 \mathrm{H}$ of Royer et al. (2010). C, Fraction of neurons responsive to nonspatial cues (e.g., fear, stress, and reward location) at dorsal and ventral hippocampus, extracted from several studies (Ferbinteanu et al., 2003; Moita et al., 2003; Royer et al., 2010). Insets show a zoom-in on the dorsal data points. For Moita et al. (2003), values were computed as the fraction of auditory-responsive neurons (above baseline). For Ferbinteanu et al. (2003), values are the fraction of journey-dependent neurons on the start arm (toward reward). For Royer et al. (2010), values were extracted from their Figure $5 \mathrm{C}$ as the fraction of goal-oriented neurons with correlation coefficient $>0.2$. D, E, Schematic illustration of possible trends along the dorsoventral axis of the hippocampus, represented on a $y$-scale from minimal to maximal values. Given the sparse experimental sampling along the dorsoventral axis (black circles), one can fit either gradual functions $(\boldsymbol{D})$ or discrete step-functions/sigmoids $(\boldsymbol{E})$, and these two possibilities result in conflicting interpretations of the nature of the representation along the dorsoventral axis as being either discrete or a gradient. To resolve this conflict, denser sampling along the dorsoventral axis will be required.

findings were interpreted by the authors as supporting the notion that theta oscillation is obligatory for spatial specificity of hippocampal place cells. However, this in- terpretation is brought into question by recent results from the dorsal hippocampus of freely behaving bats, where finely tuned place cells were shown to exist with- out a continuous theta oscillation. Moreover, the nature of theta oscillation in the bat's dorsal hippocampus was intermittent (Ulanovsky and Moss, 2007), much like the intermittent theta reported by Royer et al. (2010) in the rat ventral hippocampus. The existence of place cells without continuous theta oscillation argues against the interpretation that continuous theta oscillation is an obligatory requirement for place cell spatial tuning.

Third, Royer et al. (2010) suggested that nonspatial factors such as reward, fear, and stress robustly affect the firing of ventral, but not dorsal, hippocampal neurons. In a clever design using a seven arm maze, rats could distinguish between different maze arms by their walls. Five of the seven maze arms were "closed arms" that had $35 \mathrm{~cm}$ high walls; the other two arms were "open arms" that had no walls at all. The open arm circumstances are highly similar to an elevated platform design, often used in behavioral studies of fear, stress, and anxiety (Xu et al., 1997). Interestingly, Royer et al. (2010) found that many of the ventral, but not dorsal, neurons effectively distinguished between the open and closed arms [Royer et al. (2010), their Fig. 3], distinguished outbound (toward reward location) and inbound trajectories (away from reward location) [Royer et al. (2010), their Figs. 3, 4], and often fired as a function of distance from reward location [Royer et al. (2010), their Fig. 5]. Together, they interpreted these results as pointing to distinct representations by dorsal and ventral hippocampal neurons, suggesting that the firing patterns of ventral neurons are highly affected by nonspatial factors, whereas those of dorsal neurons primarily represent spatial locations. However, I wish to raise two main caveats to this interpretation. (1) If indeed the reason for the ventral neurons distinguishing between open and closed arms is related to fear or stress, then a behavioral correlate of stress or fear, such as increased freezing or slower velocity on the open arms compared with the closed arms, should have been quantified by the authors to support the proposed interpretation; however, no such quantification is reported in the article. In fact, because the animals were well accustomed to the behavioral tasks before recordings, it is likely that very little fear/ stress is induced by walking on the elevated open arms, which makes the stress-/fear-related interpretation less likely. (2) The encoding of nonspatial factors, such as fear, stress, and reward locations, has been previously reported also 
in dorsal hippocampal neurons (Fig. 1C). For example, dorsal neurons have been shown to acquire conditioned auditory responses in a fear-conditioning task (Moita et al., 2003) and to effectively encode reward locations and contexts (Ferbinteanu and Shapiro, 2003; Lee et al., 2006). These previous findings suggest a more subtle distinction between dorsal and ventral neurons with respect to coding for nonspatial cues.

In summary, an alternative interpretation of the results of Royer et al. (2010) can be proposed: both spatial and nonspatial information is encoded at all levels of the dorsoventral axis of the hippocampus, but the form of computation changes gradually along that axis, possibly in concert with the nature of theta oscillations (Fig. 1D). This form of organization is consistent with anatomical data suggesting that spatial information can reach even the most ventral parts, and alternatively, nonspatial information can reach the most dorsal parts via associational networks of the hippocampus or through intrinsic connections between dorsal and ventral entorhinal cortex, the main input structure of the hippocampus (Andersen et al., 2007).

Importantly, while the results of Royer et al. (2010) provide novel and valuable data on the properties of neurons in ventral hippocampus, it remains unclear whether representations change in a slow gradient along the dorsoventral axis of the hippocampus, or the dorsal and ventral hippocampus are functionally sharply distinct. Royer et al. (2010) propose distinct representations between dorsal and ventral hippocampus, which seem to contradict recent findings suggesting continuously changing spatial representations along the dorsoventral axis (Kjelstrup et al., 2008). How can this discrepancy be resolved? One explanation is that the sparse experimental sampling along the dorsoventral axis (Fig. 1D,E, black dots) can be fitted by either distinct functions (Fig. 1E, sigmoids) or by continuously changing gradients (Fig. 1D), and hence any of the competing interpretations is supported by the current data. Therefore, a key challenge to the hippocampus research community is to systematically conduct neural recordings using denser experimental sampling along the dorsoventral axis. Such studies should incorporate larger arenas and direct behavioral measures of nonspatial correlates, such as stress/fear and reward. It is then that the true nature of representation along the dorsoventral axis of the hippocampus would be revealed.

\section{References}

Andersen P, Morris R, Amaral D, Bliss T, O’Keefe J (2007) The hippocampus book. Oxford: Oxford UP.

Ferbinteanu J, Shapiro ML (2003) Prospective and retrospective memory coding in the hippocampus. Neuron 40:1227-1239.

Jung MW, Wiener SI, McNaughton BL (1994) Comparison of spatial firing characteristics of units in dorsal and ventral hippocampus of the rat. J Neurosci 14:7347-7356.

Kjelstrup KB, Solstad T, Brun VH, Hafting T, Leutgeb S, Witter MP, Moser EI, Moser MB (2008) Finite scale of spatial representation in the hippocampus. Science 321:140-143.

Lee I, Griffin AL, Zilli EA, Eichenbaum H, Hasselmo ME (2006) Gradual translocation of spatial correlates of neuronal firing in the hippocampus toward prospective reward locations. Neuron 51:639-650.

Maurer AP, Vanrhoads SR, Sutherland GR, Lipa P, McNaughton BL (2005) Self-motion and the origin of differential spatial scaling along the septo-temporal axis of the hippocampus. Hippocampus 15:841-852.

Moita MA, Rosis S, Zhou Y, LeDoux JE, Blair HT (2003) Hippocampal place cells acquire location-specific responses to the conditioned stimulus during auditory fear conditioning. Neuron 37:485-497.

O'Keefe J, Dostrovsky J (1971) The hippocampus as a spatial map. Preliminary evidence from unit activity in the freely-moving rat. Brain Res 34:171-175.

Royer S, Sirota A, Patel J, Buzsáki G (2010) Distinct representations and theta dynamics in dorsal and ventral hippocampus. J Neurosci 30:1777-1787.

Ulanovsky N, Moss CF (2007) Hippocampal cellular and network activity in freely moving echolocating bats. Nat Neurosci 10:224-233.

Xu L, Anwyl R, Rowan MJ (1997) Behavioural stress facilitates the induction of long-term depression in the hippocampus. Nature 387: 497-500. 